proportion of fat contained diminishes more rapidly than in an inverse ratio. Thus, whilst with opaque point reading of 3 c.c. we have 26.3 per cent. of fat, with I4 c.c. we have only $4 . \mathrm{I}$ per cent. of fat, and with 24 we have only 1.4 per cent.

Skim milk absorbs more light than diluted sweet milk containing the same quantity of fat as 10 to 0.268 . This is an important point, and deserves to have some attention paid to it, although it would be somewhat out of place to go into it more in detail here. I have thought it well, however, to point out some of the difficulties met with in milk analysis, and to indicate that it is only within recent years that any organised attempt has been made to overcome them.

\title{
EXPERIMENTAL RESEARCHES REGARDING THE VIRUS OF CONTAGIOUS PLEURO-PNEUMONIA OF THE OX. ${ }^{1}$
}

\section{By M. S. ARLOING, Director of the Lyons Veterinary College.}

WITH the assistance of the agricultural administration we have pursued for five years researches regarding the virus of contagious pleuro-pneumonia, with the object of discovering a more sure and practical method of preventive inoculation.

During this considerable lapse of time we have frequently had the obliging assistance of MM. Rossignol, Alexandre, Duprez, Laquerrière, Delaforge, and Robcis, who have undertaken the duty of procuring for us the means of study.

It was necessary before all to determine the virulent agent of contagious pleuro-pneumonia.

Our efforts, we believe, have been crowned with success. We are therefore constrained to give in the following paragraphs a resume of the part of our work which has conducted to that result.

\section{A.-Bacteriological Study of the Lesions of Contagious Pleuro- pneumonia of the $O x$.}

1.

Willems and Van Kempen, in I 852 , had pointed out the presence of fine molecular granulations in the exudate of the lesions of contagious pleuro-pneumonia; but the first attempt to demonstrate the real microbien nature of the virus of this disease belongs to Bruylants and Verriest of Louvain. In I880 these two experimenters thought that they had found in the lungs of affected animals a characteristic micrococcus, which they cultivated in various bouillons. They did not continue their researches, which were soon forgotten after 1882 , at which time M. Pasteur declared before the Agricultural Society of Melun that the virus of pleuro-pneumonia did not grow in our ordinary meat infusions (bonillons). Two years later, however, Poels and Nolen announced that the exudate of the diseased lung contained

1 Translated from the Journa] de Médecine Veterinaire et de Zootechnie, Ootober 1889. 
micrococci exactly similar to those which Friedländer had described in pneumonia of man. But the researches of Lustig (I885) did not confirm the preceding. Lustig isolated from the lymph of the freshly inflamed parts :-(I) A bacillus which liquefied gelatine ; (2) a micrococcus whose colonies resembled cooked white of egg; (3) another micrococcus differing from the preceding by the golden yellow colour of its cultures; (4) another micrococcus, whose culture on gelatine resembled a drop of orange wax. The last of these presented bacillary and round forms associated in such a way that its most constant character was furnished by its colour. Cornil and Babes also investigated this subject. The results which they obtained were not very precise, for they estimated in I 886 that their researches would have to be repeated, and they included in this opinion all the work of the authors who had preceded them. Such was the actual state of the question when we undertook the investigation which we proceed to describe.

\section{II.}

In the serosity which flows from the walls of a section made across a diseased lung, one perceives few microbes in comparison with the importance of the lesions; these are very short bacilli and some isolated micrococci, disjoined or associated in pairs.

If one distributes the serosity which escapes spontaneously fron a section among a number of flasks containing bouillon, the majority of these remain sterile. The cultures are almost certainly negative if they have been inseminated with clear serosity carefully aspirated from the depth of the lesions with a fine pipette. Probably this was the modus faciendi followed by the Melun Commission. To obtain fertile cultures it is necessary to deposit in a flask a large quantity of seed, or rather to use serosity which escapes from the section under the influence of scraping.

But these cultures contain several microbes which it is necessary to isolate.

In place of proceeding to the dilution and fractional insemination of cultures, it is more simple, in view of the rarity of the microbes, to distribute directly the pulmonary serosity on nutritive gelatine by the aid of a platinum needle or a fine pipette. From colonies arising in this way four different microbes are obtained:-(I) A bacillus which promptly and completely liquefies the gelatine; (2) a non-liquefying micrococcus whose white colonies resemble drops of wax; (3) a micrococcus of which the white colonies spread in a thin layer, which as it grows older become wrinkled and folded; (4) another micrococcus whose elongated or circular colonies take a beautiful yelloworange tint. It seems to us certain that Lustig has isolated the first two, but he must have observed under numbers 3 and 4 a mixture of our numbers 2,3 , and 4 .

We propose to call the first the Pneumobacillus liquefaciens bovis, the second Pneumococcus gutta-cerei, the third Pneumococcus lichenoindes, the fourth Pnenmococcus flavescens. It is possible that the Pn. guttacerei and the Pn. flavescens respond to the Microc. cereus albus and Microc. cereus flavus of Passet; we have not been able to assure ourselves of this by comparison. 


\section{III.}

A certain number of characters warrant the assertion that these are indeed four different species of microbes. Thus the first two are facultatively xrobic and anærobic; the last two are exclusively rerobic; the pneumobacillus vegetates only at $+3.5^{\circ} \mathrm{C}$.; pneumococcus lichenoides does not develop on potato, while the three others grow vigorously on it. But it is well to know that they offer some rather curious examples of polymorphism. The pneumobacillus, which in bouillon is very short, sometimes almost round, becomes elongated, slightly increased in sizc, and regular on gelatine. The pneumococcus gutta-cerei increases in size, and the pueumococcus lichenoides becomes pseudo-bacillary on the solid media.

IV.

The micrococci are almost always combined with the pneumobacillus in greater or smaller numbers in all points of the freshly inflamed pulmonary lesions, and even in the sequestra. Despite this coexistence, it is impossible to attribute to the four microbes an equal part in the genesis of pleuro-pneumonia. It is probable, on the contrary, that one of the species constitutes the essential agent of the virus. We determine that in the following note.

\section{B.-Determination of the Causal Microbe of Contagious Pleuro- pneumonia of the $O x$.}

I.

The determination of a pathogenic microbe is complete when one has reproduced by inoculation with its cultures the lesions from which it proceeds. Such determination is difficult to effect in the case of pleuro-pneumonia, seeing that hitherto, save in rare exceptions, one has not succeeded in reproducing the disease in the ox by intrapulmonary inoculation of the natural virus, or by any other method. The experimenters have simply provoked locally in the loose subcutaneous connective tissue a hard slowly growing tumour, accompanied often in young animals by metastatic synovitis. This tumour almost infallibly causes death by the products which the specific microbe secretes in its substance, and which the subject gradually absorbs. If the animal survives it becomes almost indifferent to new inoculations-it possesses immunity.

We ought then to strive to reproduce with the before-described cultures of microbes either the above-mentioned subcutaneous lesions or the pulmonary lesions; but, after this short preamble, it will be foreseen that we have not attained this object without having surmounted several obstacles.

Lustig having obtained by a first inoculation of his orange microbe a small transitory subcutaneous tumour, and by a second inoculation a still less voluminous tumefaction, believed himself warranted in comparing these insignificant effects to those of inoculation with the pulmonary serosity, and in regarding this microbe as the agent which excites pleuro-pneumonia. 
II.

In our opinion the specific microbe is the pneumobacillus liquefaciens bovis. We have determined that as follows:-

If one inoculates half a centimetre cube of the culture of each of these microbes which we have found into different points of the loose connective tissue of a young bullock or heifer, one obtains at each point a flat, œdematous, hot, and painful tumefaction, which, save a rather persistent nodule, disappears in five or six days. The most extensive tumefaction is produced by the pneumobacillus; then come in order of intensity the tumours caused by the pneumococcus gutta-cerei, the pneumococcus flavescens, and the pneunococcus lichenoides. If one inoculates several successive generations, a moment arrives at which the pnezmobacillus alone produces a local effect. Inoculated into the lung, the trachea, or the veins, the same cultures have not excited pleuropneumonia.

We are far from the subcutaneous effects of the fresh pulmonary serosity; but all the microbes which are contained in that, isolated or associated, immediately lose the greater part of their special activity when cultivated. Nevertheless, it will be remarked that the pneumobacillus liquefaciens is that whose cultures produce the phenomena most analogous to those of the fresh virulent serosity. That is an argument in favour of our hypothesis, and it will become more convincing if we add that the pneumobacillus is found in every affected lung, while the three other microbes are sometimes absent, that it exists also in the metastatic synovites which develop remote from the subcutaneous tumour, and finally, that the general effect of its products of secretion and of the filtered pulmonary serosity are similar.

\section{III.}

These various considerations have led us to try whether the inoculation of pure cultures of a more energetic pneumobacillus would give more satisfactory results.

We have observed that the special activity of the pulmonary serosity is increased when its microbes are made to evolve under the skin of the healthy ox. In afterwards isolating the liquefying bacillus contained in the subcutaneous tumour, one has some chances of obtaining more active organisms than by taking them directly from the lung. The cultures that we have made in this manner have been subsequently inoculated ( $I$ ) into the lung and pleura, and (2) into the veins of the ox.

When injected into a lung, $4 \mathrm{cc}$. immediately produced trembling, acceleration of the respiration, cough, and elevation of temperature. A month afterwards there were found at the autopsy villous false membranes on the visceral pleura, and some foci of chronic pneumonia disseminated through both lungs.

In the veins $20 \mathrm{cc}$. determined the death of two young cattle of $200-250$ kilogr. in ten hours, after a series of very remarkable accidents. At the autopsy there was found marked congestion of the lungs; all the interlobular spaces were infiltrated with serosity, as in the recent lesions of pleuro-pneumonia. Eight cc. injected in two doses, with an 
interval of some days, caused immediate accidents analogous to the preceding but attenuated, and to these succeeded some dyspnoea and intermittent digestive disturbance; during the first days blood streaked with mucus flowed from the nose. If the subjects are killed at the end of a month there is found swelling of the bronchial and mediastinal lymphatic glands; there spread through both lungs rosy or whitish alterations, with small pseudo-purulent nuclei, corresponding to the lesions which authors assign to the chronic forms of epizootic pleuro-pneumonia.

It is not doubtful, notwithstanding the rapid march of the process, that we have inoculated the pathogenic agent of pleuro-pneumonia. Let us give a supplementary demonstration by way of counter-proof.

IV.

We have injected, within thirty-six hours, into the jugular of a young bullock, I 2 cc. of the natural serosity containing the reinforced virus. The animal presented some days later an unusual but very important localisation. The autopsy, made twenty-six hours after inoculation, showed that the lesions of the interlobular and sub-pleural spaces of the pleuro-pneumonic lung were developed in the external crural region. Moreover, a great number of inoculations on gelatine made with the serosity of these lesions gave only colonies of the pneumobacillus liquefaciens.

Thus it seems to us demonstrated, as far as it can be done in the meantime, that we know the conditions which determine a pulmonary localisation, that the pueumobacillus liquefaciens is indeed the essential living element of the virus of contagious pleuro-pneumonia of the ox.

\title{
TRAUMATIC PERICARDITIS AND ITS ACCOMPANYING MORBID PROCESSES. ${ }^{1}$
}

\author{
By Veterinary Surgeon Bongartz, Bonn, Germany.
}

A consideration of this frequent disease of cattle ought to claim a general interest, alike on account of its peculiar insidious course, the importance of its pathological anatomy, and its forensic significance. We see it arise most frequently in the neighbourhood of the larger towns, in the small dairies which accept the kitchen refuse, potatoparings, fragments of greens, etc., out of the town, in order to use these as food for the cows. As is known, these people, in order to obtain a higher yield of milk, are in the habit of administering to their cows a large quantity of warm liquid, containing roots, potato-parings, refuse of greens, with the addition of bran, brewer's grains, bread, etc. In such kitchen refuse there is not seldom found needles of various sizes, hair-pins, broken knitting-needles, nails, and the like, which are ingested with the food, and play their rôle as excitants of a series of morbid processes. The circumstance that in all the districts near this town 